\title{
ARTICLE
}

\section{Conservative Evaluation of Remaining Radioactivity on Decommissioned Nuclear Power Plant Sites}

\author{
Tadao TANAKA ${ }^{1 *}$, Taro SHIMADA ${ }^{1}$, Takeshi ITO², Takahiro HIRANO ${ }^{3}$, \\ Takenori SUKEGAWA ${ }^{1}$ \\ ${ }^{1}$ Japan Atomic Energy Agency, Tokai, Naka, Ibaraki 319-1195, Japan \\ ${ }^{2}$ Japan ATOX Co.,Ltd., Tokai, Naka, Ibaraki 319-1112, Japan \\ ${ }^{3}$ HOKKO Electronics Co.,Ltd.,Furano, Sorachi, Hokkaido 071-0502, Japan
}

\begin{abstract}
Nuclear facility sites after decommissioned are necessary to confirm to be decontaminated prior to be released from nuclear safety regulations. A conservative method to evaluate radioactivity remaining on the sites of decommissioned nuclear power plants was proposed in this study, and its validity was discussed. Cesium 137 was taken up to validate the evaluation method as one of the typical radionuclides for nuclear power plants. In the evaluation method concept, all of the Cs-137, which are in reality distributed across the area of interest, is assumed to be the single point source located at the furthest position on the ground surface of the area from a detector. In such a configuration, most conservative result was given by Monte Carlo calculations, and the detectable time (time need to detect enough amount of radioactivity) predicted by the calculation was approximately equal to the actual in-situ measurement time by the potable pure-germanium semiconductor detector. These results indicate that the proposed evaluation method was reasonable for the conservative evaluation of remaining radioactivity.
\end{abstract}

KEYWORDS: decommission, nuclear power plant, remaining radioactivity, cesium 137, in-situ measurement

\section{Introduction}

According to the basic Japanese nuclear policy, nuclear power plant sites are allowed to be released from nuclear safety regulations after the plants are decommissioned. The sites are necessary to confirm to be decontaminated prior to be released $^{1}$. In the present study, we proposed a conservative method to evaluate radioactivity remaining on the sites of decommissioned nuclear power plants, and confirmed validity of the evaluation method by in-situ tests.

The in-situ measurement with a portable pure-germanium semiconductor detector (Ge detector) was applied to detect a very low radioactivity remaining on the sites. The Ge detector does not need preprocessing and waiting for attenuation, can provide measured data in real time on the sites, and can distinguish radionuclides by the intrinsic emission energies. The in-situ method has also has advantages such as being capable of enlarging the range of the object, and being able to secure representativeness of the data obtained in the sites ${ }^{1), 2)}$.

Cesium-137 was taken up to validate the evaluation method as one of the typical radionuclides for nuclear power plants.

\section{Concept of the Evaluation Method}

As basic assumption in the evaluation method, Cs-137 pollution is attributable to ground surface deposition, and no significant Cs-137 pollution is presumed to be existence in an interest area, from the history investigation on the decommissioned nuclear power plant site.

The concept of the proposed method is drawn in Fig. 1.

*Corresponding Author, E-mail:tanaka.tadao26@jaea.go.jp

(C) Atomic Energy Society of Japan
All of the Cs-137, which are in reality distributed across the area of interest, are assumed to present as the single point source located at the furthest position on the ground surface of the area from a detector. In such a configuration, the counting time need to detect the Cs-137 point source (the detectable time) is predicted using Monte Carlo simulation. If radiation from the Cs-137 point source is not detected for longer than the predicted detectable time, it can be proven that the radioactivity remaining in the interest area is lower than the radioactivity corresponding to the assumed Cs- 137 point source most difficult to detect. The difference from previous method is that it is not necessary to measure the absolute radioactivity.

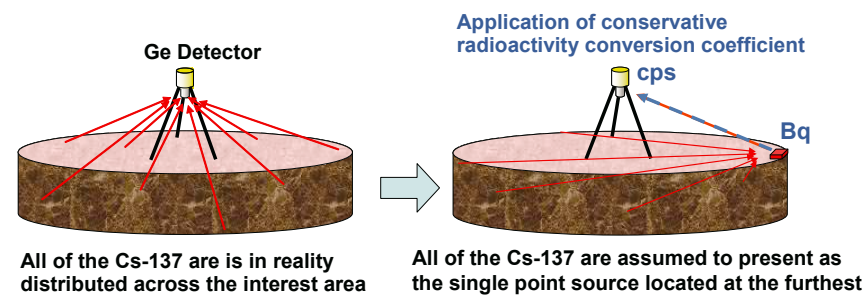

Fig. 1 Concept of the conservative evaluation method.

\section{Reasonability of the Evaluation Concept}

In the evaluation concept, it is premise that the detectable time predicted by the calculation is identical with measurement time to detect a radiation source actually. So the validity of the detectable time predicted in a configuration system was confirmed by the field tests. 


\section{Field tests}

Figure 2 shows a schematic view of field tests. To avoid the influence of radiation from various artificial structures the tests were carried out around the center of large space (site area of $18,000 \mathrm{~m}^{2}$ ), and to avoid the influence of radiation from fallout origin by past nuclear weapons testing, the disturbed site, where the soil layer had been dug up many times, was selected.

Two standard point sources of different radioactivity of $0.29 \mathrm{MBq}$ and $0.44 \mathrm{MBq}$ were prepared as Cs-137 point sources. The point source was sealed in a column type polyethylene container of $5 \mathrm{~cm}$ in diameter and $5 \mathrm{~cm}$ in height. The point source was placed at a fixed distance $(3 \mathrm{~m}$ - $20 \mathrm{~m}$ ) from the Ge detector (Refer to Fig. 2). The Ge detector was set up at $1 \mathrm{~m}$ in height above the ground level. The Cs-137 point source of lower radioactivity $(0.29 \mathrm{MBq})$ was used for shorter distance measurement $(3,5,7$ and 10 $\mathrm{m})$, and that of higher radioactivity $(0.44 \mathrm{MBq})$ was for longer distance measurement, as summarized in Table 1.

The German regulatory standard of Cs-137 radioactivity for site release is $0.06 \mathrm{~Bq} / \mathrm{g}^{3)}$. If Cs-137 of $0.06 \mathrm{~Bq} / \mathrm{g}$ was uniformly distributed over the depth of $1 \mathrm{~cm}$ from ground surface in a soil layer of $1.6 \mathrm{~g} / \mathrm{cm}^{3}$ in density, there is 0.30 $\mathrm{MBq}$ of $\mathrm{Cs}-137$ in the interest area of $10 \mathrm{~m}$ in radius. The radioactivity of the Cs-137 point source used in this study was as much as that calculated from the German regulatory standard. It seems that the radioactivity in Table 1 is suitable level for studies on the site release.

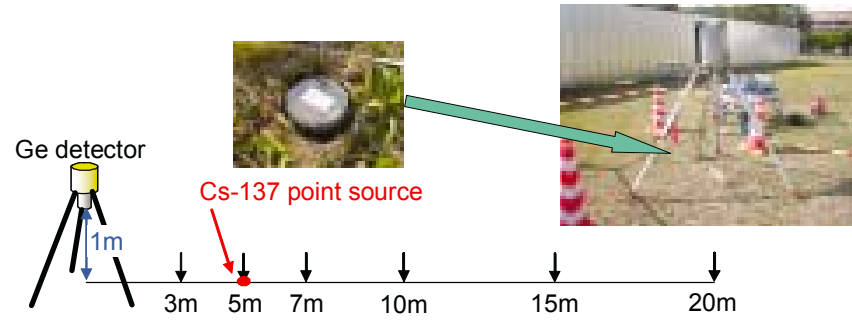

Fig. 2 Schematic view of field tests.
The time-dependent changes of the detection limit counting rate and net counting rate at the peak of $661.7 \mathrm{keV}$ were analyzed. The peak counting rate from background radioactivity for $12,000 \mathrm{sec}$ was $0 \mathrm{cpm}$. The actual measurement time was defined to be the time interval where the peak counting rate was greater than the detection limit counting rate.

The time courses of the detection limit counting rate and the peak count rate, which were measured for the configuration of "20 m in distance from detector", are shown in Fig. 3, as a typical example. The measurements were carried out triplicate runs under the same testing condition.

\section{Prediction of the detectable time for radiation source set up at the furthest position}

The prediction procedure of the detectable time for radiation source set up at the furthest position is explained about Fig. 3. Relationship between detection limit counting rate $N_{\mathrm{L}}$ (cps) and measurement time $t$ (s) can be approximated to Eq. (1).

$$
t=a \times N_{L}^{-b}
$$

Substituting the detection limit counting rate with minimum radioactivity $A_{\mathrm{L}}(\mathrm{Bq})$ should be detected and radioactivity conversion coefficient $K(\mathrm{~Bq} / \mathrm{cps})$, we obtain

$$
t=a \times\left(A_{L} / K\right)^{-b}
$$

The $K$ corresponding to configurations in Fig. 1 and Fig. 2 was calculated from the particle transport simulation using the Electron-Gamma Shower (EGS)-5 code ${ }^{4)}$. Theoretical minimum measurement time that the peak counting rate was greater than the detection limit counting rate for the Cs-137 point source can be predicted by using $A_{\mathrm{L}}$ and $K$ in each testing condition.

Table 1 Measurement distance and Cs-137 point source.

\begin{tabular}{|l|c|c|c|c|c|c|}
\hline Distance from Ge detector & $3 \mathrm{~m}$ & $5 \mathrm{~m}$ & $7 \mathrm{~m}$ & $10 \mathrm{~m}$ & $15 \mathrm{~m}$ & $20 \mathrm{~m}$ \\
\hline Cs-137 point source & $0.29 \mathrm{MBq}$ & $0.29 \mathrm{MBq}$ & $0.29 \mathrm{MBq}$ & $0.29 \mathrm{MBq}$ & $0.44 \mathrm{MBq}$ & $0.44 \mathrm{MBq}$ \\
\hline
\end{tabular}

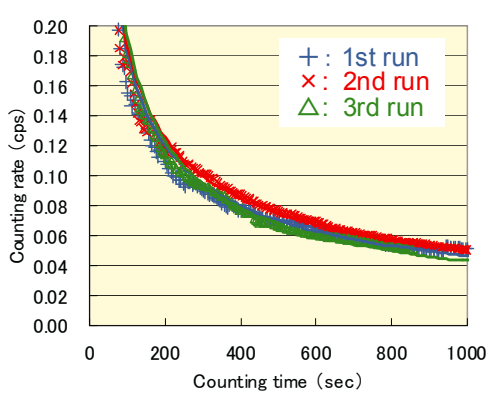

(a) Time courses of detection limit counting rate
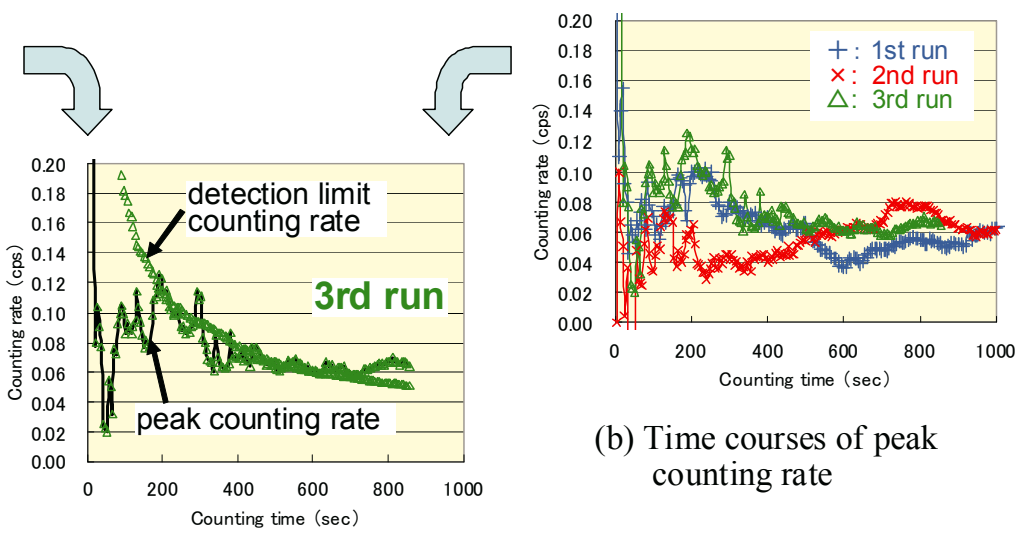

(b) Time courses of peak counting rate

Fig. 3 Time courses of detection limit counting rate and peak counting rate at $661.7 \mathrm{keV}$. 
As been obvious from Fig. 3 (a), the curves in the three runs were not the same. Therefore, $a$ and $b$ in Eq. (1) and Eq. (2) contain uncertainty. From the maximum value and the minimum value of $a$ and $b$ decided by fitting Eq. (2) to all the measurement results, the uncertainties of $a$ and $b$ were decided to $9.91 \pm 4.49,1.43 \pm 0.32$, respectively. Thus uncertainty in the detectable time predicted is expressed by

$$
t=(9.91 \pm 4.49) \times\left(A_{\mathrm{L}} / K\right)^{-(1.43 \pm 0.32)}
$$

\section{Measurement of actual time detected radiation source set up at the furthest position}

The measurement time that the Cs-137 point source set up at the furthest position was regarded to be detected, when the peak counting rate was greater than the detection limit counting rate, was decided by according to Fig. 4. As shown in Fig. 4, point $\mathrm{A}$ is measurement time that the peak counting rate exceeded firstly the detection limit counting rate, and $\mathrm{B}$ is measurement time that the peak counting rate was to exceed always the detection limit counting rate. The $\mathrm{A}$ and $\mathrm{B}$ can be assumed to be judgment values for the actual measurement time detected the Cs-137 point source.

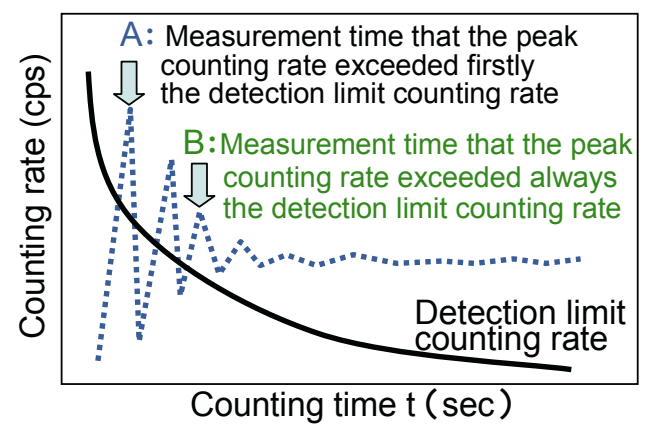

Fig. 4 Judgment concept for actual measurement time.

Uncertainty in the detectable time predicted from Eq. (3) and the actual measurement time $\mathrm{A}$ and $\mathrm{B}$ were summarized in Table 2. The differences between A and B were large in some testing conditions. It is necessary to consider large error in the measurement time. The predicted detectable time was roughly equal to the actual measurement time, by considering large error in the measurement precision. The results suppose that the prediction procedure of the detectable time for radiation source set up at the furthest position was reasonable.

Table 2 Uncertainty in the detectable time predicted by calculation and actual measurement time.

\begin{tabular}{|c|c|c|c|c|c|c|}
\hline \multirow{2}{*}{ Detection time } & \multicolumn{6}{|c|}{ Distance from Ge detector } \\
\hline & $3 \mathrm{~m}$ & $5 \mathrm{~m}$ & $7 m$ & $10 \mathrm{~m}$ & $15 \mathrm{~m}$ & $20 \mathrm{~m}$ \\
\hline Predicted detactable time (sec) & $4-11$ & 3-34 & $8-73$ & $62-223$ & $101-269$ & $182-484$ \\
\hline $\begin{array}{l}\text { Actual measurment time (sec) } \\
\text { 1st run (A-B) } \\
\text { 2nd run (A-B) } \\
\text { 3rd run (A-B) } \\
\text { 4th run (A-B) }\end{array}$ & $\begin{array}{l}15-15 \\
10-10 \\
15-15 \\
10-10\end{array}$ & $\begin{array}{l}30-30 \\
20-20 \\
15-15 \\
20-20\end{array}$ & $\begin{array}{l}65-150 \\
155-265 \\
300-300 \\
110-250\end{array}$ & $\begin{array}{l}465-530 \\
300-300 \\
370-370 \\
237-370\end{array}$ & $\begin{array}{c}590-725 \\
785-960 \\
740-1310\end{array}$ & $\begin{array}{l}210-775 \\
635-635 \\
190-515\end{array}$ \\
\hline & & $15-15$ & $210-210$ & $110-110$ & & \\
\hline
\end{tabular}

$\mathrm{A}$ is measurement time that the counting rate at the peak exceeded firstly the detection limit counting rate, and $B$ is measurement time that the counting rate was to exceed always the detection limit counting rate, in Fig. 4.

\section{Conservativeness of the Evaluation Concept 1. Radioactivity conversion coefficient}

The radioactivity conversion coefficient $K$ is obviously influenced by the vertical distribution because of the shielding effect with the soils.

Radioactive substances discharged from the nuclear power plants distribute into the surrounding soil layers, where the radioactive concentration decreases exponentially from the ground surface. The vertical distribution tendency is described by the depth distribution parameter $\beta^{5 \text { ), }}$

$$
A(Z)=A_{0} \cdot \exp (-Z / \beta),
$$

where $A(Z)$ represents radioactive concentration $(\mathrm{Bq} / \mathrm{g})$ at $Z$ $\mathrm{cm}, A_{0}$ surface radioactive concentration $(\mathrm{Bq} / \mathrm{g}), Z$ depth $(\mathrm{cm})$, and $\beta$ depth distribution parameter $\left(\mathrm{g} / \mathrm{cm}^{2}\right)$.

For example, relationship between $\beta$ and the distribution of Cs-137 concentration was illustrated in Fig. 5. In general, vertical distribution of radioactive substances in soil layers is often evaluated with $\beta=4.8 \mathrm{~g} / \mathrm{cm}^{2), 5), 6)}$, when the radioactive pollution is attributable to the surface deposition.

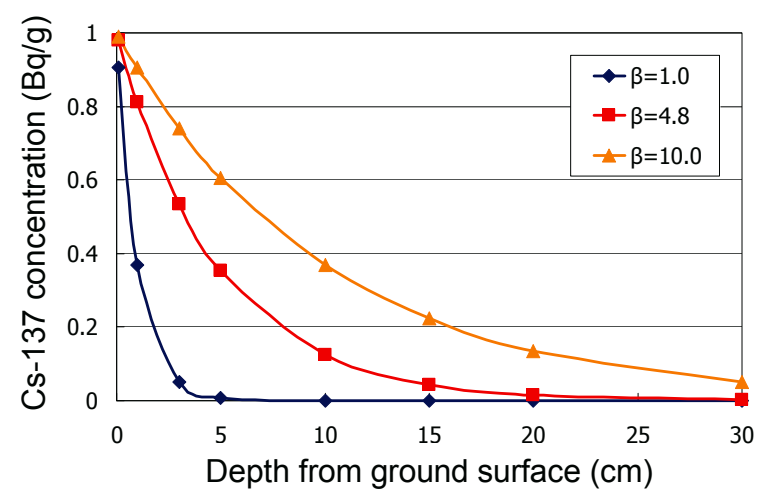

Fig. 5 Relationship between $\beta$ and vertical distribution of Cs-137 concentration.

The conservativeness of the radioactivity conversion coefficient in the proposed evaluation method was discussed from the viewpoint of distribution tendency of radioactive substances. The radioactivity conversion coefficients were compared between the three cases; case (i) Cs-137 assumed to be uniformly distributed, case (ii) Cs-137 assumed to be exponentially distributed, and case (iii) Cs-137 assumed to be the single point source located at the furthest position. The radioactivity conversion coefficient was calculated by using the EGS-5 code under the conditions in Table 3. In the calculation, the radius and the depth of the interest area, and the $\beta$ value were varied. Figure 6 shows the results of comparing the radioactivity conversion coefficients $K$ of each case at intervals of $1,3,5$, and $10 \mathrm{~m}$ in radius. The $K$ of the case (iii) Cs-137 assumed to be the single point source was drawn as the horizontal line. Most $K$ of the case (iii) are larger than that of the case (i) and the case (ii), except for a part of the result of $1 \mathrm{~m}$ in radius, and the case (iii) was the most conservative configuration. The results mean that the radioactivity remaining in the interest area may be conservatively evaluated by setting the evaluation unit to 3 
$\mathrm{m}$ or more in radius.

\section{Influence of radiation from outside area}

The Ge detector also counts the radiation from outside of interest area. According to some previous knowledge, contribution from outside of the interest area of $10 \mathrm{~m}$ in radius is assumed for $15 \%$, when Cs- 137 is distributed with $\beta=4.8 \mathrm{~g} / \mathrm{cm}^{2}$ from the surface of an infinite plane area ${ }^{6)}$. The influences of radiation from the outside are increased with decreasing radius of the interest area. Thus, the radioactivity evaluated using the calculated radioactivity conversion coefficient is to provide more conservative values in actual situation.

\section{Conclusion}

The conservative method to evaluate radioactivity remaining on the sites of decommissioned nuclear power plants was proposed. The in-situ measurement test using the portable Ge detector proved that the proposed evaluation method was reasonable for the conservative evaluation.

At the next stage, it is necessary to discuss on considerations for fallout and natural radionuclides, on requirements for detection lower limit, and on optimizations of concrete approving procedures and measurement conditions.

\section{Acknowledgment}

This work is a part of study funded by Japan Nuclear Energy Safety Organization. The authors wish to express their appreciation to Dr. Shinichi Nakayama, and $\mathrm{Mr}$. Masayuki Mukai, Japan Atomic Energy Agency, for valuable discussions.

\section{References}

1) USNRC, Multi-agency Radiation Survey and Site Investigation Manual (MARSSIM), NUREG-1575, Rev.1, U. S. Nuclear Regulatory Commission (USNRC), (2000).

2) A. N. Tyler, "In situ and airbone gamma-ray spectrometry", in Ed. P. P. Povinec, Analysis of Environmental Radionuclides, Elsevier, Hungary, 407 (2008).

3) SSK, Recommendation of the German Commission for Radiation Protection as of 24. 04. 98, German Commission on Radiological Protection (SSK), Bonn, 1998.

4) H. Hirayama, Y. Namio, A. F. Bielajewa, S. J. Wildermana, W. R. Nelson, The EGS5 Code System, KEK Report 2005-8, High Energy Accelerator Research Organization (KEK), (2005).

5) ICRU, Gamma-Ray Spectrometry in the Environments, ICRU Report 53, International Commission on Radiation Units and Measurements (ICRU), (1994).

6) MEXT, In-situ Measurement Method by Using Germanium Semiconductor Detector, Radioactivity Measurement Method Series No. 33, Ministry of Education, Culture, Sports, Science and Technology (MEXT), Tokyo, (2008), [in Japanese].
Table 3 Conditions in Monte Carlo calculation by EGS-5 code. Number of photon generation $10^{8}-10^{9}(661.7 \mathrm{keV})$ Height of detector $1 \mathrm{~m}$ Soil density $1.6 \mathrm{~g} / \mathrm{cm}^{3}$ Ge crystal $\phi 61,45.5 \mathrm{~mm}$, Efficiency $30 \%$ Configuration types of $\mathrm{Cs}-137$ radiation source

(i) for single point source located at the furthest position

(ii) for uniform distribution

(iii) for exponential distribution $(\beta=1,4.8,10)$

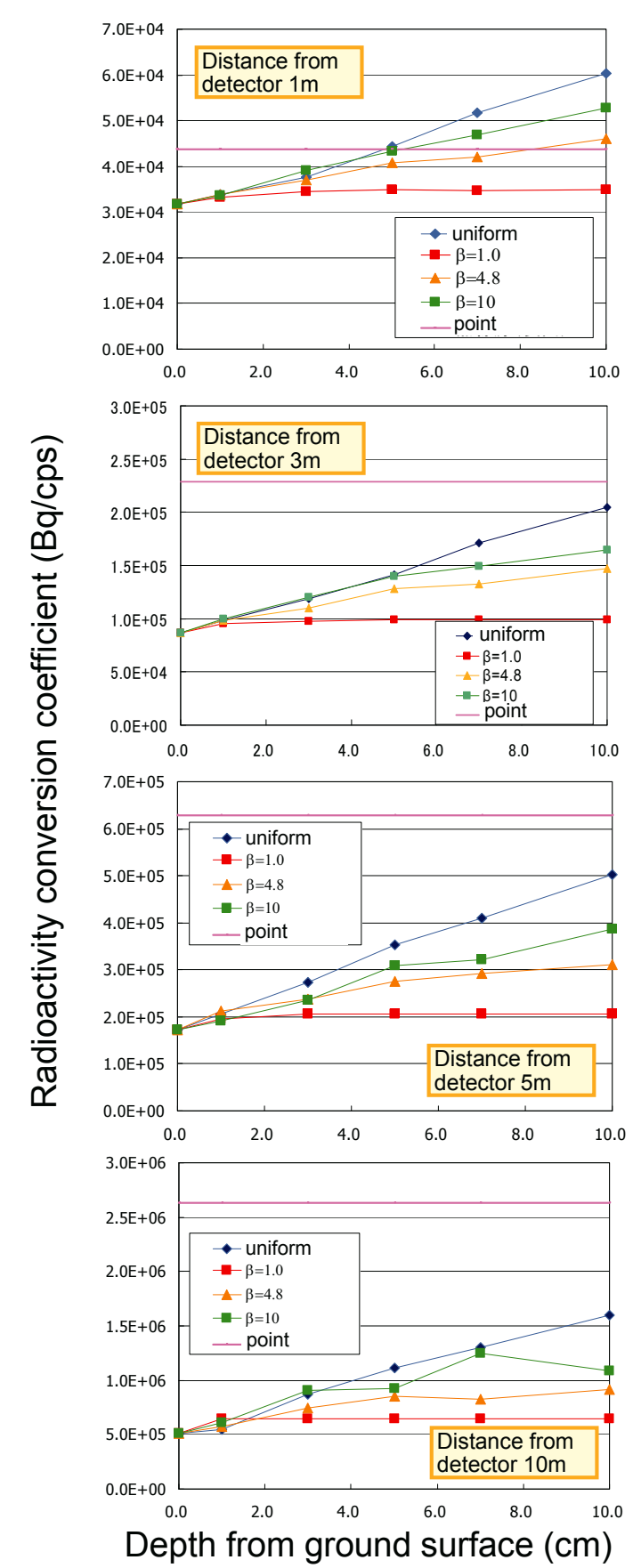

Fig. 6 Comparison of radioactivity conversion coefficients calculated at intervals of $1,3,5$, and $10 \mathrm{~m}$ in radius. 